\title{
Efeito do tamanho do fragmento na dispersão de sementes de Copaíba (Copaifera langsdorffii Delf.)
}

\author{
Ananza Rabello ${ }^{1}$, Flávio Nunes Ramos $^{1}$ \& Érica Hasui ${ }^{1,2}$ \\ ${ }^{1}$ Laboratório de ecologia de fragmentação - Ecofrag, Departamento de Ciências Biológicas e da Terra, \\ Universidade Federal de Alfenas - UNIFAL, \\ Rua Gabriel Monteiro, 700, CEP 37130-000 Alfenas, MG, Brasil \\ ${ }^{2}$ Autor para correspondência: Érica Hasui, e-mail: ericahasui@yahoo.com
}

RABELLO, A., RAMOS, F.N. \& HASUI, E. Effect of fragment size on Copaifera langsdorffii seeds dispersal. Biota Neotrop. 10(1): http://www.biotaneotropica.org.br/v10n1/en/abstract?article+bn00810012010.

\begin{abstract}
Effect of fragment size on Copaifera langsdorffii seed dispersal. Habitat fragmentation may cause functional disruption in seed dispersal, if the disperser species become locally extinct in the fragments. However during the fragmentation process, there is empirical evidence of replacement of specialist by generalist species, and these generalist species could also replace the functional activity of the specialists in the ecosystem. We studied bird frugivory on $C$. langsdorffii in order to evaluate the patch size effect on the potential of seed dispersal of this plant. During 2008 we chose 20 focal trees distributed in five Atlantic forest remnants (range 29.2 to 104.8 ha), looking for which bird species consumed the fruits, describing their foraging behavior and consequently evaluating the potential for seed dispersal. The results suggest that there was lower similarity of bird richness among the patches. The ability to disperse seeds also varied among birds and their patterns of distribution and abundance were related with patch size. The number of good disperser species and their interactions were positively related with patch size $(\mathrm{R} 2=0.85 ; \mathrm{p}=0.05)$; in contrast, the poor dispersers had the opposite tendency $(\mathrm{R} 2=0.77$; $\mathrm{p}=0.08$ ). Therefore the preservation of larger patch size increases the interaction events between C. langsdorffii and their good dispersers, and consequently improving their fitness.
\end{abstract}

Keywords: birds, Atlantic Forest, fragmentation, plant-animal interaction, foraging tactics.

RABELLO, A., RAMOS, F.N. \& HASUI, E. Efeito do tamanho do fragmento na dispersão de sementes de Copaíba (Copaifera langsdorffii Delf.). Biota Neotrop. 10(1): http://www.biotaneotropica.org.br/v10n1/pt/ abstract?article+bn00810012010.

Resumo: A fragmentação de habitats pode afetar negativamente a relação mutualística entre plantas e animais, se estes tornarem-se localmente extintos nos fragmentos. No decorrer do processo de fragmentação há evidências de substituição de espécies especialistas por generalistas, adotando também sua função no ecossistema. Nós estudamos a frugivoria de Copaifera langsdorfii por aves para avaliar o efeito do tamanho do fragmento no potencial de dispersão das suas sementes. Durante o ano de 2008 foram selecionadas vinte plantas focais distribuídas em cinco remanescentes de Mata Atlântica (amplitude 29,2 a 104,8 ha) para descrever o comportamento de forrageamento de aves consumidoras e avaliar o potencial de dispersão de sementes de cada espécie através do método de observação focal. O potencial de dispersão variou entre as espécies, juntamente com o padrão de distribuição e abundância nos diferentes fragmentos. O tamanho dos fragmentos afetou positivamente o número de interações de espécies com bom potencial de dispersão $(\mathrm{R} 2=0,85 ; \mathrm{p}=0,05)$. A riqueza de espécies com má qualidade de dispersão foi negativamente afetada pelo tamanho do fragmento, mas com significância marginal $(\mathrm{R} 2=0,77$; $\mathrm{p}=0,08)$. Portanto a preservação de fragmentos de maior tamanho garante mais eventos de interação entre a C. langsdorfii e seus bons dispersores, e consequentemente favorece seu sucesso reprodutivo.

Palavras-chave: aves, fragmentação, interação animal planta, Mata Atlântica, táticas de forrageamento. 


\section{Introdução}

A extinção de animais dispersores de sementes devido ao processo de fragmentação florestal pode afetar negativamente as plantas zoocóricas (Cordeiro \& Howe 2003). Isto é agravado em florestas neotropicais, onde aproximadamente $90 \%$ das espécies dependem dos vertebrados frugívoros para dispersar as suas sementes (Herrera 2002). Esta dependência das plantas está relacionada em alguns casos ao aumento da sobrevivência das sementes e ao estabelecimento da plantas jovens a alguma distância da planta-mãe, já que nas redondezas da planta-mãe há uma intensa predação por insetos e mamíferos. Somando-se a isto há uma alta competição pelos recursos com a própria planta-mãe (Fonseca \& Antunes 2007).

Neste contexto, já foi comprovado que a fragmentação pode limitar o potencial de aves frugívoras para dispersão de sementes, representando assim uma barreira para a regeneração da mata (Pizo \& Vieira 2004). Muitas espécies de aves que vivem no interior da floresta sentem-se ameaçadas pela predação e não conseguem atravessar nem mesmo faixas estreitas de ambiente aberto. Com isto, espécies de plantas com frutos carnosos ou sementes aderentes também são afetadas, porque dependem desses animais para dispersar as suas sementes (Primack \& Rodrigues 2001). O resultado é que fragmentos isolados tendem a receber (de outras áreas) e dispersar menos sementes como conseqüência das ameaças que os animais dispersores sofrem com a fragmentação (Jordano et al. 2006).

O fato de várias espécies dispersoras não conseguirem deslocarse em habitats fragmentados gera distúrbios na sua interação com as plantas, pois a capacidade para dispersar as sementes por longas distâncias pode ser um fator chave na sobrevivência de espécies da flora no local (Ozinga 2004, Jordano et al. 2006). Quanto maior a distância entre os fragmentos, mais isolados eles serão (se a matriz apresentar baixa permeabilidade), aumentando assim a probabilidade das espécies vegetais tornarem-se localmente extintas, e diminuindo a probabilidade de reconstrução dessa área degradada (Ozinga 2004).

Outro aspecto da dispersão de sementes relacionado com a fragmentação envolve alterações no tamanho dos fragmentos, o que pode gerar profundos efeitos na interação ecológica planta-animal. A perda de habitat pode levar ao declínio de espécies de aves frugivoras especialistas, que apresentam uma alimentação altamente restrita, consistindo em animais suscetíveis à extinção (Hasui 1994). Por outro lado, espécies generalistas beneficiam-se com este processo pela diminuição de competição pelos frutos. A substituição desses frugívoros pode afetar a qualidade da dispersão das sementes e as taxas de estabelecimento das plantas jovens (Kiriba et al. 2008). Em fragmentos maiores pode haver a presença de dispersores com maior qualidade e diversidade para dispersão, enquanto que a diminuição no tamanho do habitat pode causar diferenças no tipo de dispersor, prejudicando a dispersão das sementes das plantas (Mustajärvi 2001). Por esses fatores, vê-se que a fragmentação não é apenas um proces- so de diminuição de espaço, mas sim um no qual muitos impactos biológicos indiretos são observados em virtude de desdobramentos dos efeitos iniciais (Ewers \& Didham 2006).

Deste modo, este estudo avaliou os efeitos do tamanho e do grau de isolamento dos fragmentos na dispersão de sementes de Copaifera langsdorffii pelas aves, analisando o potencial de dispersão de sementes de cada espécie. C. langsdorffii foi escolhida por ser fonte de alimento para muitas espécies de aves, e por estar presente nos remanescentes da região. A previsão é que a riqueza de espécies de aves frugívoras com alta qualidade de dispersão seja positivamente relacionada com tamanho do fragmento e negativamente com seu isolamento. O inverso é previsto para as espécies de aves com baixa qualidade de dispersão.

\section{Material e Métodos}

\section{1. Área de estudo}

Os fragmentos distribuem-se no município de Alfenas, no sul de Minas Gerais (Tabela 1). A região encontra-se dentro do domínio da Mata Atlântica, na Floresta Estacional Semidecidual, com altitude média de $880 \mathrm{~m}$. Os remanescentes apresentam clima tropical moderado úmido, com temperatura anual em torno de $23{ }^{\circ} \mathrm{C}$ e inverno de dois a quatro meses secos e déficit hídrico pequeno, entre 10 e $30 \mathrm{~mm}$, sendo seu regime de captações médias 1400 a 1700 mm (Costa 1998). A paisagem encontra-se em estado de grande fragmentação com aproximadamente $4 \%$ de cobertura vegetal nativa, sendo a matriz composta por plantações de café, de cana e pasto (Fundação SOS Atlântica \& Instituto Nacional de Pesquisas Espaciais, 2009). Em termos de vegetação, o município encontra-se na área de transição entre os biomas Mata Atlântica e Cerrado (Oliveira \& Ferreira 2008), porém com a vegetação remanescente encontrando-se extensamente alterada pelo homem.

A análise preliminar de uma paisagem de $30 \mathrm{~km}$ de raio ao redor de Alfenas foi realizada através de imagens de satélite (CBERS), com resolução de $20 \mathrm{~m}$ com o auxílio do programa ArcGis 9.2. A classificação da imagem serviu para identificação dos fragmentos florestais em estágio avançado de sucessão. Posteriormente, foram calculados o tamanho e o índice de proximidade dos fragmentos (métrica de proximidade, PROX) com o uso do Fragstats (McGarigal et al. 2002), sendo selecionados cinco fragmentos ao longo de um gradiente de tamanho (amplitude 29,2 a 104,8 ha; Tabela 1), de acordo com a presença de $C$. langsdorffii em frutificação e com a logística de deslocamento entre os fragmentos.

O índice de proximidade é considerado uma medida de descontinuidade do ambiente que leva em conta a área que esta mancha engloba. Ele está altamente relacionado com a capacidade dispersiva dos organismos e esta capacidade, por sua vez, está relacionada com a área disponível das manchas adjacentes. Neste trabalho, as análises das manchas adjacentes foram consideradas dentro de um buffer de

Tabela 1. Descrição das métricas de mancha (tamanho e isolamento da mancha através do índice de proximidade, PROX, McGarigal et al. (2002)) para os cinco fragmentos florestais amostrados em Alfenas, MG.

Table 2. Description of patch metrics (patch size and isolation by proximity index, PROX, McGarigal et al. (2002)) for the five forest patches sampled in Alfenas, MG.

\begin{tabular}{|c|c|c|c|}
\hline Fragmentos & Coordenadas Geográficas & Área (ha) & Índice de proximidade \\
\hline 1 & $46^{\circ} 00^{\prime} 21^{\prime \prime} \mathrm{O}$ e $21^{\circ} 22^{\prime} 34^{\prime \prime} \mathrm{S}$ & 29,18 & 68,81 \\
\hline 2 & $46^{\circ} 08^{\prime} 06^{\prime \prime}$ O e $21^{\circ} 25^{\prime} 20^{\prime \prime} \mathrm{S}$ & 42,80 & 155,80 \\
\hline 3 & $46^{\circ} 09^{\prime} 33^{\prime \prime}$ O e $21^{\circ} 26^{\prime} 33^{\prime \prime} \mathrm{S}$ & 65,20 & 68,81 \\
\hline 4 & $45^{\circ} 58^{\prime} 39^{\prime \prime}$ O e $21^{\circ} 30^{\prime} 05^{\prime \prime} \mathrm{S}$ & 85,49 & 58,42 \\
\hline 5 & $45^{\circ} 51^{\prime} 31^{\prime \prime}$ O e $21^{\circ} 19^{\prime} 52^{\prime \prime} \mathrm{S}$ & 104,80 & 0,00 \\
\hline
\end{tabular}


$1 \mathrm{~km}$ ao redor de cada mancha-alvo. O programa repete o cálculo para cada mancha dentro do buffer envolvendo tanto a área quanto as distâncias das manchas adjacentes com a mancha-alvo, de acordo com a Equação 1:

$$
\operatorname{Prox}_{\mathrm{i}}=\sum_{\mathrm{s}=1}^{\mathrm{N}} \frac{\mathrm{a}_{\mathrm{j}}}{\mathrm{d}_{\mathrm{ij}{ }^{2}}}
$$

onde:

Prox $\mathrm{i}_{\mathrm{i}}$ é o isolamento da mancha-alvo i (considera todas as manchas dentro de uma região de determinado tamanho - buffer);

$A_{i j}$ : área da mancha $\mathrm{j}$;

$\mathrm{D}_{\mathrm{ij}}$ : distância entre a mancha i e a mancha $\mathrm{j}$.

O índice de proximidade é adimensional e, considerado o inverso, porque o isolamento diminui com seu aumento. Isto ocorre porque, como a área é dividida pela distância ao quadrado, quanto maior a distância entre duas manchas de florestas de mesmo tamanho, menor será o valor do índice e maior o isolamento. Por outro lado, manchas de floresta que diferem quanto ao tamanho, mas estão a uma mesma distância, terão índices maiores quanto maior a área (Bender et al. 2003).

\section{Espécie vegetal}

Copaifera langsdorffi Desf. é uma planta da família Fabaceae, conhecida popularmente como Copaíba, com 10 a 15 m de altura. Está amplamente distribuída no Brasil, sendo encontrada desde o estado do Amazonas até as regiões nordeste, sudeste, centro-oeste e sul (Lorenzi 2000). É uma planta decídua ou semidecídua, encontrada principalmente na floresta latifoliada da bacia do Paraná. Ocorre tanto em floresta primária como em formações secundárias, sendo característica das formações de transição do cerrado para Floresta Estacional Semidecidual (Lorenzi 2000).

Copaifera langsdorffii floresce durante os meses de dezembro a março. Seus frutos amadurecem em agosto e setembro, com a planta quase totalmente despida da folhagem, e seu tamanho e pesos são em média, $2,43 \times 1,94 \mathrm{~cm}$ e 3,46 g, respectivamente (20 indivíduos, 100 frutos). Produz anualmente grande quantidade de sementes de cor marrom, envolta parcialmente por arilo alaranjado amplamente consumido e disperso pelas aves (Pedroni et al. 2002). Esta espécie foi escolhida por apresentar frutos abundantes e ser de fácil identificação.

\section{Potencial de Dispersão e Padrão de Resposta à Fragmentação}

A avaliação da eficiência dos dispersores nos fragmentos foi realizada por meio de observação focal, que consiste na permanência do observador por 30 minutes próximo à planta-mãe, registrando o comportamento alimentar dos frugívoros visitantes (Galetti et al. 2003). As observações foram realizadas em 20 indivíduos de C. langsdorffii (quatro por fragmento), e com distância mínima de $30 \mathrm{~m}$ para aumentar a independência das observações (Develey 2003). O frag. 1 apresentou menor quantidade de C. langsdorffii com a distância mínima de $30 \mathrm{~m}$, sendo possível trabalhar com apenas quatro indivíduos e essa quantidade foi padronizada nos outros fragmentos. Cada indivíduo foi amostrado por quatro vezes, totalizando 40 horas de amostragens, nos períodos de agosto e setembro de 2008 (época predominante da frutificação). As observações foram realizadas no período da manhã (7:00 às 9:00), horário em que as aves preferencialmente visitam as plantas em frutificação.

Durante as observações focais foram anotados: espécie de ave visitante, número de indivíduos dessa espécie, horário da visita, tempo de permanência na planta, número de frutos consumidos, tempo empregado na alimentação e detalhes sobre a forma de manipulação do alimento. As visitas somente foram registradas para os indivíduos que manipularam pelo menos um diásporo. Sempre que um indivíduo abandonou temporariamente a planta-mãe ou mudou a forma de coleta de fruto, se procedeu a contagem de uma nova visita (Guimarães 2003).

Quando vários indivíduos de uma mesma espécie estiveram ao mesmo tempo visitando a planta, a atenção da observação fixou-se sobre um único indivíduo (Cullen 2003). A identificação das espécies de aves foi realizada com auxílio de guias de campo (Develey \& Endrigo 2004), sendo que em todas as observações foi utilizado um binóculo. As espécies foram caracterizadas quanto à guilda alimentar (frugívoros, onívoros ou insetívoros), através da descrição da dieta alimentar das espécies presentes em Sick (2001), e em relação a sua dependência do habitat florestal, através da base de dados contida em Stotz et al. (1996): alta (exclusivamente do interior de floresta), média (habitat florestal, mas com ocorrência na borda de mata), média baixa (habitat florestal, mas ocorrência em borda, capoeira e campina) e baixa (preferência por áreas abertas, como cerrado, pastagem e agricultura).

\section{Forrageamento}

Segundo Pascotto (2006), a coleta e a manipulação dos frutos pelas aves acontecem de forma variada. Com isso, o presente trabalho qualificou a forma de manipulação do diásporo, enfocando seu potencial para a dispersão. Esses procedimentos foram necessários para distinguir a ação dos dispersores e, por conseguinte, o sucesso reprodutivo da planta (Volpato \& Mendonça-Lima 2002; Machado \& Rocha 2005).

\section{Bom potencial de dispersão}

- Engolir (EI) = a ave engoliu o diásporo inteiro sem qualquer movimentação da mandíbula, pairado no ar ou empoleirado na planta-mãe.

- Segurar (SD) = a ave coletou, mas não consumiu imediatamente. O diásporo ficou preso entre as pontas das mandíbulas, sendo carregado para longe da planta-mãe.

\section{Mau potencial de dispersão}

- Esmagar $(E D)=$ a ave espremeu ou movimentou o diásporo entre as mandíbulas para retirar a semente, descartando-a sob a planta-mãe.

- $\operatorname{Picar}(\mathrm{PA})=$ a ave bicou e removeu apenas um pedaço do arilo. A semente permaneceu ligada à planta-mãe, sem qualquer evidência de dispersão.

Nas relações que foram estabelecidas com o tamanho e isolamento dos fragmentos, os eventos de interação foram agrupados em função da qualidade no potencial de dispersão (boa ou má), sendo posteriormente divididos pelo tempo despendido na alimentação. Isto porque quanto maior o tempo de permanência da ave, maiores serão as chances das sementes caírem sob a planta-mãe, comprometendo o sucesso de dispersão.

\section{Análise dos Dados}

A partir dos eventos de interação foram realizadas estimativas de riqueza de espécies de aves frugivoras em interação com C. landgsdorfii para cada fragmento, utilizando o método de rarefação por indivíduo (Krebs 1989). Isto permitiu realizar comparações da riqueza de frugívoros entre os fragmentos, padronizadas pelo mesmo número de interações. A similaridade na composição de frugívoros entre os fragmentos foi calculada através do índice de Jaccard. Posteriormente foi examinada a correlação entre esta similaridade e a distância entre os fragmentos por meio da Correlação de Sperman. Para avaliar o efeito da fragmentação sobre o potencial de dispersão 
de sementes, as variáveis independentes de tamanho do fragmento e de isolamento foram previamente transformadas $(\log 10)$ a fim de obter a normalidade dos dados e homogeneidade nas variâncias. Em seguida, foram realizadas regressões lineares simples onde o número de eventos de interação e riqueza de espécies foram classificados de acordo com a qualidade do potencial de dispersão, determinada a partir do comportamento de forrageio dos dispersores. Após os cálculos de regressões, foram avaliados os resíduos com inspeção visual dos gráficos dos erros, e conseqüente remoção de um outlier.

\section{Resultados}

\section{Riqueza de espécies e qualidade de dispersão}

Foram identificadas 13 espécies de aves que consumiram os diásporos de $C$. langsdorffii, sendo apenas uma (Ramphastos toco Statius Muller) classificada como frugívora. Quanto ao habitat preferencial, nenhuma das espécies apresentou alta preferência florestal; ao contrário, a maioria dos habitats das espécies foi caracterizada pela alta diversidade de formações vegetais (floresta, capoeira, cerrado e campina) e tolerância à interferência antrópica (borda de floresta, culturas agrícolas e pastagens). Thraupis sayaca Linnaeus foi a mais abundante e comum a todos os fragmentos, porém considerada como má dispersora por comer somente o arilo, descartando a semente sob a planta-mãe. Por outro lado, Elaenia spp. apresentou a maior qualidade de dispersão, em termos de freqüência nos fragmentos, forma de manipulação e consumo de diásporos (Tabelas 2 e 3).

Os fragmentos variaram em composição das espécies, apresentando baixa similaridade entre eles (índice de Jaccard, média $=0,52 \pm 0,27$ ), principalmente nas comparações com o Frag. 5, cujos índices de similaridade foram inferiores a 0,50 . Não foi observada uma correlação entre a similaridade e distância geográfica entre os fragmentos amostrados (Coeficiente de Sperman = 0,25, p =0,49).

Em termos de riqueza de espécies houve diferenças significativas entre os fragmentos, com um número menor de espécies nos Frag. 3 e 5 (Figura 1). Pelas inclinações de suas curvas de rarefação, não existem evidências de acréscimo de novas espécies, caso haja um aumento no número de interações observadas. Os demais fragmentos apresentaram riquezas similares, mas provavelmente tenham uma riqueza de espécies acima do observado.

\section{Relação entre fragmentação e potencial de dispersão.}

Nas regressões lineares simples realizadas com todos os fragmentos não foram observadas relações entre a riqueza de espécies, número de interações/hora com as métricas da paisagem (tamanho e grau de isolamento dos fragmentos; $p>0,05)$. As análises dos resíduos das regressões e as inspeções visuais dos gráficos dos erros mostraram um comportamento muito discrepante do Frag. 5 (Figura 2), estando fora dos padrões definidos pelas outras observações, o que contribuiu muito nos resíduos (outlier). Ao excluí-lo, em todas as análises, observou-se que o tamanho dos fragmentos afetou a qualidade de dispersão das sementes de C. langsdorffii. O número de interações/hora com boa qualidade de dispersão foi positivamente relacionado com o tamanho do fragmento. A riqueza de espécies com má qualidade de dispersão também foi negativamente afetada pelo tamanho do fragmento, mas com significância marginal (Figura 3). Quanto ao efeito do isolamento dos fragmentos, não foi identificado qualquer relação com o potencial de dispersão ou com a riqueza de espécies dispersoras (Tabela 4).

Tabela 2. Descrição do comportamento de forrageamento das espécies que consumiram diásporos de C. langsdorffi durante 40 horas de observação focal em cinco fragmentos amostrados em Alfenas, MG.

Table2. Foraging behavior of bird species that consumed C. langsdorffii diaspores during 40 hours of focal plant sampling in five patch samples in Alfenas, MG.

\begin{tabular}{|c|c|c|c|c|c|}
\hline Espécie & $\mathbf{N}^{0}$ de visitas & $\begin{array}{l}\mathrm{N}^{0} \text { diásporos } \\
\text { consumidos }\end{array}$ & $\begin{array}{c}\text { Número de } \\
\text { interação/tempo }\end{array}$ & Forrageamento $^{1}$ & $\begin{array}{l}\text { Qualidade de } \\
\text { dispersão }^{2}\end{array}$ \\
\hline Thraupis sayaca (Linnaeus, 1766) & 32 & 54 & 0,43 & $\mathrm{PA}$ & Má \\
\hline Schistochlamys ruficapillus (Vieillot, 1817) & 1 & 1 & 0,33 & $\mathrm{PA}$ & Má \\
\hline Tangara cayana (Linnaeus, 1766) & 7 & 8 & 0,5 & PA & Má \\
\hline Cyanocorax caeruleus (Vieillot, 1818) & 7 & 12 & 0,30 & $\mathrm{PA}$ & Má \\
\hline Dacnis cayana (Linnaeus, 1766) & 6 & 6 & 0,46 & PA, ED & Má \\
\hline Myiozetetes similis (Spix, 1825) & 2 & 4 & 1 & SD & Boa \\
\hline Serpophaga subcristata (Vieillot, 1817) & 7 & 8 & 0,5 & SD & Boa \\
\hline Elaenia spp. & 28 & 41 & 0,5 & $\mathrm{SD}, \mathrm{EI}$ & Boa \\
\hline Ramphastos toco Statius Muller, 1776 & 11 & 30 & 0,46 & EI & Boa \\
\hline Turdus rufiventris Vieillot, 1818 & 8 & 16 & 0,57 & EI & Boa \\
\hline Turdus amaurochalinus Cabanis, 1850 & 5 & 9 & 0,42 & EI & Boa \\
\hline Mimus saturninus (Lichtenstein, 1823) & 1 & 2 & 0,5 & EI & Boa \\
\hline Turdus leucomelas Vieillot, 1818 & 3 & 6 & 0,6 & EI & Boa \\
\hline
\end{tabular}

${ }^{1}$ Forrageamento - modo de coleta e manipulação do diásporo: Engolir $(\mathrm{EI})$ = a ave engoliu o diásporo inteiro sem qualquer movimentação das maxilas, pairado no ar ou empoleirado na planta-mãe; Segurar $(\mathrm{SD})$ = a ave coletou, mas não consumiu imediatamente. O diásporo ficou preso entre as pontas das maxilas, sendo carregado para longe da planta-mãe; Esmagar $(E D)=$ a ave espremeu ou movimentou o diásporo entre as maxilas para retirar a semente, descartando-a sob a planta-mãe; Picar $(\mathrm{PA})=$ a ave bicou e removeu apenas um pedaço do arilo. A semente permaneceu ligada à planta-mãe, sem qualquer evidência de dispersão. ${ }^{2}$ Qualidade de dispersão - baseada no comportamento de forrageamento: Má = má qualidade para o modo de coleta e manipulação PA e ED; Boa = boa qualidade para o modo de coleta e manipulação SD e EI

'Foraging behavior - attack and handling techniques of the diaspore: Gulp $(E I)=$ The bird swallowed intact diaspore without any noticeable manipulation other than being held briefly by bill, sally-hovering or perching in the parent plant; Hold (SD) $=$ the bird collected the diaspore, but did not eat immediately. The diaspore was held by bill, being carried farther from the parent plant; Mash (ED) = The bird crushed the diaspore by bill to separate and discard the seed under the parent plant, Bite $(\mathrm{PA})=$ The bird bit and removed a peace of arillus. The seed remained connected to the parent plant, without seed dispersal evidences. ${ }^{2}$ Dispersal quality - based on foraging behavior: Bad = bad quality, considering the attack and handling techniques PA and ED; Good = good quality, considering the attack and handling techniques SD and EI. 
Efeito da fragmentação na dispersão de sementes

Tabela 3. Espécies observadas consumindo os diásporos de C. langsdorffi em cinco fragmentos florestais amostrados em Alfenas, MG.

Table 3. Bird species that consumed C. langsdorffii diaspores in five patch samples in Alfenas, MG.

\begin{tabular}{|c|c|c|c|c|c|c|c|}
\hline Espécies & Guilda $^{1}$ & Dependência Florestal $^{2}$ & Frag. 1 & Frag. 2 & Frag. 3 & Frag. 4 & Frag. 5 \\
\hline Ramphastos toco & FRU & Média-baixa & 2 & 3 & 5 & 1 & - \\
\hline Thraupis sayaca & ONI & Média-baixa & 8 & 5 & 7 & 5 & 7 \\
\hline Tangara cayana & ONI & Baixa & 1 & - & 4 & - & 2 \\
\hline Dacnis cayana & ONI & Média-baixa & 4 & - & - & - & 2 \\
\hline Turdus rufiventris & ONI & Média & - & 1 & - & 7 & - \\
\hline Turdus amaurochalinus & ONI & Média & 2 & - & 3 & - & - \\
\hline Turdus leucomelas & ONI & Média & - & - & - & 3 & - \\
\hline Mimus saturninus & ONI & Média & - & - & - & 2 & - \\
\hline Myiozetetes similis & ONI & Média & - & 2 & - & - & - \\
\hline Serpophaga subcristata & INS & Média & - & 1 & 2 & 4 & - \\
\hline Elaenia spp. & INS & Baixa & 3 & 6 & 4 & 4 & 11 \\
\hline Cyanocorax caeruleus & INS & Média-baixa & - & 4 & - & - & 3 \\
\hline Shistochlamys ruficapillus & INS & Média-baixa & 1 & - & - & - & - \\
\hline
\end{tabular}

${ }^{1}$ Guilda - Classificação das aves pela preferência alimentar feita através da descrição de Sick (2001): FRU= frugívoros; ONI= onívoros; INS= insetívoro. ${ }^{2}$ Dependência florestal - classificação das aves de acordo com as características do tipo de formação vegetal do habitat, feita através da base de dados contida em Stotz et al. (1996): Alta = exclusivamente de interior de floresta; Média = habitat florestal, mas com ocorrência em borda de mata; Média baixa = habitat florestal, mas com ocorrência em borda, capoeira e campina; Baixa = preferência área aberta, cerrado, pastagem e agricultura;

${ }^{1}$ Guild - bird classification according their food preference based on Sick (2001) description: FRU= frigivorous; ONI= omnivorous; INS= insectivorous. ${ }^{2}$ Forest dependence - bird classification followed the Stotz et al. (1996) description for the habitat specialization in forest ecosystems: Alta $=$ restrict to forestinterior; Média = forest habitat, but also found at edges; Média baixa = forest habitat, but also found at edges, in secondary-growth scrub and grassland; Baixa $=$ preference in open habitats, cerrado, pastures/agricultural lands.

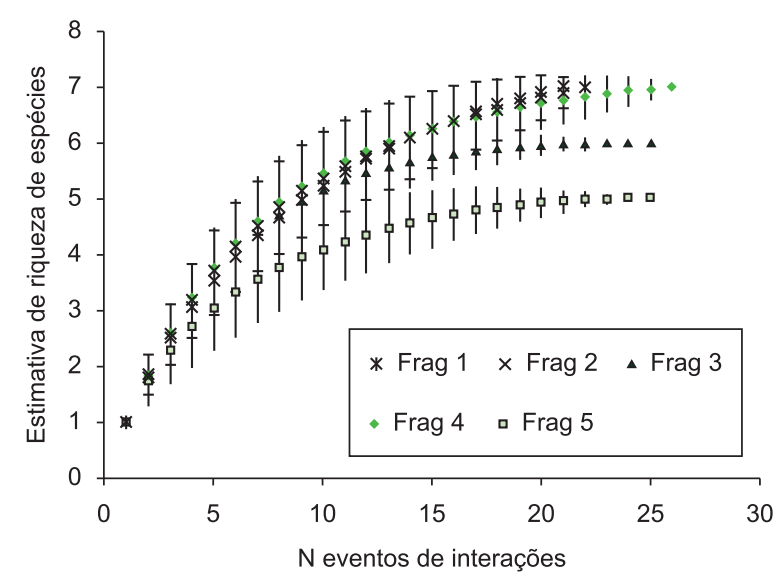

Figura 1. Curvas de acumulação médias de riqueza de espécies de aves que consumiram diásporos de C. langsdorffii em cinco fragmentos amostrados em Alfenas, MG.

Figure 1. Average of number of bird species accumulation curves that consumed diaspores of $C$. langsdorffii on five patch sampled in Alfenas, MG.

\section{Discussão}

\section{Variação na composição e riqueza de espécies entre os fragmentos}

De modo geral os resultados sugerem variações entre os fragmentos em termos de riqueza e a intensidade das interações entre C. langsdorffii e as aves. Primeiro foi verificado uma baixa similaridade na composição de espécies visitantes e muita variação no número de interações entre os fragmentos, sendo que a maioria das espécies apresentou um consumo restrito ou intensificado em poucos fragmentos. Segundo, os fragmentos menores apresentaram uma

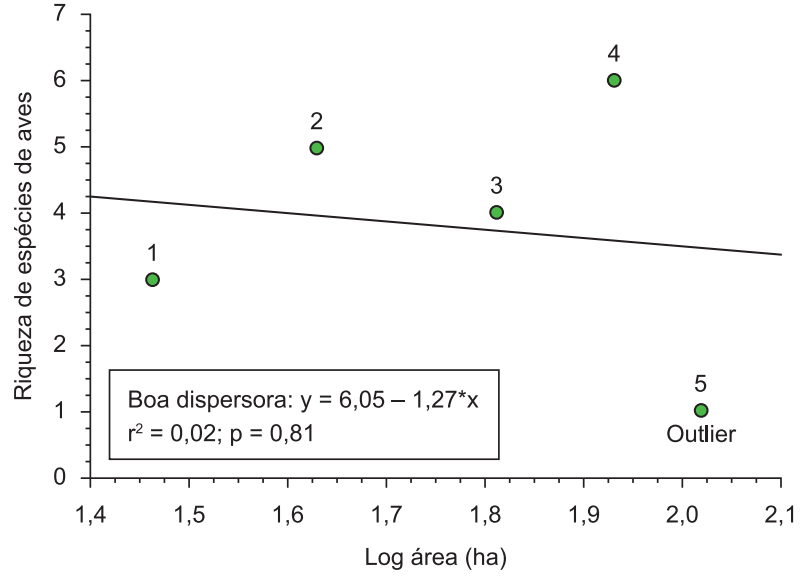

Figura 2. Relação entre o tamanho do fragmento e o potencial de dispersão de sementes de $C$. langsdorffii com boa dispersão para a exibição gráfica do outlier (ponto $5=$ Frag 5 ). $\mathrm{O}$ número ao lado do ponto representa o nome dos fragmentos.

Figure 2. Relationship between patch size and seed dispersal potential of $C$. langsdorffii with good dispersal to show the outlier (point $5=$ Frag 5). Number besides the points represents each fragment's name.

riqueza de espécies consumidoras superior ao maior e mais isolado fragmento (Frag 5). Estas variações podem ser decorrentes da ação de diversos processos que atuam em diferentes escalas. Em termos locais, diferenças de diversidade, distribuição espacial ou de proporção das espécies zoocóricas nos fragmentos podem modificar a atratividade de C. langsdorffii como fonte de alimento às aves (Price et al. 1999, Galetti \& Pizo 1996). Além disto, dependendo da intensidade e do grau de sincronia da frutificação das espécies zoocóricas, as visitas aos indivíduos de $C$. langsdorffii podem variar conforme a disposição espacial dos indivíduos em frutificação nas proximidades, e de acordo com as preferências alimentares das aves (Bronstein 1995). Ao nível de paisagem, a grande heterogeneidade ambiental pode levar a baixa 

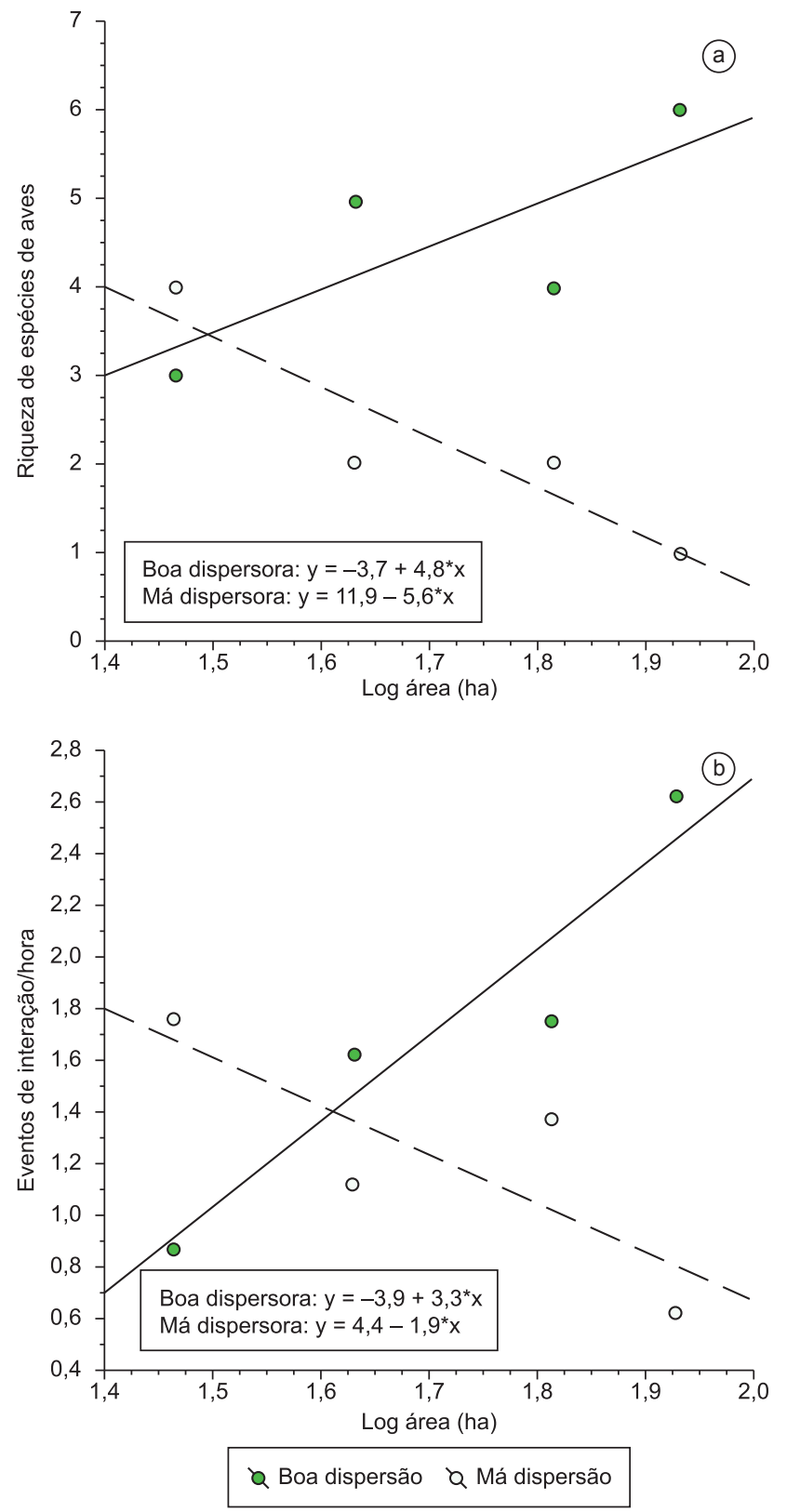

Figura 3. Efeito do tamanho do fragmento sobre o potencial de dispersão de sementes de $C$. langsdorffii. As aves foram classificadas através do comportamento de forrageio, sendo posteriormente agrupadas pelo potencial de dispersão de sementes (boa e má dispersão). a) Relação entre a riqueza de espécies de aves e o tamanho da área do fragmento. b) Relação entre o número de interações/hora e o tamanho da área do fragmento (log área).

Figure 3. Patch size effect on the seed dispersal potential of $C$. langsdorffii. The bird species were classified by their foraging behavior, and then grouped according the potential for seed dispersal (good and bad seed dispersal). a) Relationship between bird richness species and patch size (log area). b) Relationship between the number of interaction/hours and the patch size (log area).

similaridade entre os fragmentos, sendo que esta independe da distância geográfica dos fragmentos amostrados (Santos 2004).

A ausência de frugívoros com alta dependência florestal reflete o nível de degradação dos remanescentes de Mata Atlântica na região (composta por cerca de $4 \%$ da cobertura original), pois espécies desta guilda são mais sensíveis à perda de habitat, sendo mais propensas à extinção em pequenos remanescentes (Watson et al. 2004). As espé-
Tabela 4. Coeficientes $\left(\mathrm{r}^{2}\right)$ das regressões lineares simples obtidos nas relações entre tamanho e isolamento dos fragmentos (variáveis independentes) com a riqueza de espécies e número de interações/hora de aves que consumiram diásporos de $C$. langsdorffii (variáveis dependentes) em quatro fragmentos florestais amostrados em Alfenas, MG. As aves foram classificadas de acordo com o comportamento de forrageio, sendo posteriormente agrupadas pelo potencial de dispersão de sementes (boa ou má dispersão). Análise com exclusão do outlier.

Table 4. Simple linear regression coefficients for patch size and isolation (independent variables) on the bird richness and the number of interaction/ hours of birds that consumed C. langsdorffii diaspores (dependent variables) in four forest fragments in Alfenas, MG. The bird species were classified by their foraging behavior, and then grouped according to the potential for seed dispersal (good and bad seed dispersal). Analysis with outlier exclusion.

\begin{tabular}{lcc}
\hline \multicolumn{1}{c}{ Variáveis } & $\mathbf{R}^{\mathbf{2}}$ & $\mathbf{P}$ \\
\hline Riqueza boa X Log Área & 0,38 & 0,23 \\
Riqueza má X Log Área & 0,77 & 0,08 \\
Interação boa X Log Área & 0,85 & 0,05 \\
Interação má X Log Área & 0,51 & 0,18 \\
Riqueza boa X Isolamento & 0,02 & 0,84 \\
Riqueza boa X Isolamento & 0,00 & 0,95 \\
Interação boa X Isolamento & 0,03 & 0,82 \\
Interação má X Isolamento & 0,00 & 0,97 \\
\hline
\end{tabular}

cies frugívoras tendem a ser substituídas por aves mais generalistas, tanto em termos alimentares quanto em relação à restrição ao tipo ou qualidade do habitat (Cordeiro \& Howe 2003, Ribon et al. 2003).

A substituição por espécies mais generalistas já foi registrada em outras áreas tropicais fragmentadas (Feeley \& Terborgh 2006) e essa permuta pode ter consequências drásticas para o sucesso reprodutivo de C. langsdorffii, pelo elevado número de eventos de interações de má qualidade de dispersão de sementes. Isto pode estar comprometendo a manutenção local da população, por consequiência da alta concentração espacial de indivíduos aparentados próximo à planta-mãe. Este tipo de distribuição espacial pode gerar um alto grau de endogamia nas gerações futuras (Ramos et al. 2007). Cordeiro \& Howe (2003) mostraram que as taxas de dispersão de sementes e recrutamento de plantas jovens nas redondezas da planta-mãe são maiores em áreas fragmentadas em comparação a florestas contínuas, devido à redução na dispersão por falta de dispersores como aves. Em termos do funcionamento do ecossistema, a interferência prejudicial no processo de dispersão de sementes pode reduzir a capacidade de estabelecimento de muitas espécies de plantas, interrompendo o equilibro dinâmico ou o processo de sucessão natural do ecossistema, como observado em florestas contínuas (Moran et al. 2008).

\section{Fragmentação e potencial de dispersão}

O potencial de dispersão de sementes de C. langsdorffii é afetado qualitativa (quanto maior a riqueza de espécies dispersoras, maior é a diversidade de comportamento) e quantitativamente pelo tamanho do fragmento. Os fragmentos maiores tenderam a um número mais elevado de interações/hora e riqueza de espécies de aves dispersoras. Outros estudos também demonstraram os efeitos diretos da fragmentação sobre as interações mutualísticas ou agonísticas (Garcia \& Chacoff 2006).

Para as aves dispersoras com alta mobilidade, como Ramphastos toco, o uso complementar dos fragmentos como fonte de alimento deve ser freqüente pela distribuição heterogênea (espacial e temporal) dos frutos ao longo do ano (Bronstein 1995). No entanto, os fragmentos menores devem ser menos atrativos e menos freqüentados, porque proporcionalmente, devem oferecer menos frutos (Prince et al. 1999). 
Esse fator merece atenção pelo fato da dispersão de sementes e as interações entre dispersor - planta determinarem a estrutura espacial, dinâmica e composição das populações e comunidades de plantas como a C. langsdorffii (Spiegel \& Nathan 2007).

Contrário às expectativas originais, não foi obtida relação entre o potencial de dispersão de $C$. langsdorffii e o grau de isolamento dos fragmentos. A relação era esperada pelas restrições que as espécies florestais apresentam em se locomover pela matriz. No entanto, todas as espécies registradas são generalistas quanto ao tipo de habitat (média e baixa dependência florestal) e, por isto, são menos exigentes em relação ao isolamento dos fragmentos. Neste caso, a seleção destes fragmentos provavelmente não foi adequada para identificar o efeito do isolamento. Paisagens com uma maior porcentagem de cobertura vegetal original e com uma variação maior no grau de isolamento poderiam garantir a presença de espécies visitantes com alta dependência florestal e, assim permitir a identificação do efeito do isolamento. Porém, essa é a situação da região e provavelmente de muitas outras, sendo de extrema importância entender a dinâmica regional para a preservação de populações vegetais e animais.

No Brasil foram realizados muitos trabalhos com frugivoria e /ou dispersão de sementes com aves fazendo inferência sobre o potencial dispersor de uma determinada espécie e suas relações com a fragmentação florestal (e.g. Krugel et al. 2006). Porém poucos estudos analisaram as consequências do tamanho dos fragmentos dentro deste processo mutualístico animal-planta. Por isso, são necessários trabalhos futuros que analisem este efeito utilizando um maior número de fragmentos e com maior amplitude de variação, além de mais espécies zoocóricas e outros grupos de animais. Isto irá proporcionar um conhecimento mais claro sobre a interferência do tamanho do fragmento no processo de dispersão de sementes e consequentemente sobre a restauração de ambientes degradados.

Portanto, este estudo mostrou que existe diferença na composição e distribuição das espécies de aves dispersoras entre os diferentes tamanhos de fragmentos, comprovando que o tamanho do fragmento está relacionado com a boa qualidade de dispersão e riqueza de espécies de aves boa dispersoras. Assim, a preservação de tamanhos maiores de fragmentos aumenta os eventos de interação entre a C. langsdorffii e seus bons dispersores, favorecendo o seu sucesso reprodutivo e estabelecendo a sobrevivência tanto das aves quanto da população dessa espécie vegetal.

\section{Agradecimentos}

Agradecemos a todos que acompanharam esta pesquisa: Gregório R. Menezes, Rodolpho C. Rodrigues, Diego G. S. Pereira e Hugo S. Pereira por colaborarem com o desenvolvimento deste trabalho. Ao grupo de pesquisa ECOFRAG e a FAPEMIG pelo apoio financeiro. Agradecemos, por fim, à Unifal-Mg que nos cedeu o transporte aos fragmentos e a permissão concedida pelos proprietários dos fragmentos.

\section{Referências}

BENDER, D.J., TISCHEBDORF, L. \& FAHRIG, L. 2003. Using patch isolation metrics to predict animal moviment in binary landscapes. Landsc. Ecol. 18(1):17-39.

BRONSTEIN, J.L. 1995. The plant-pollinator landscape. In Mosaic landscapes and ecological processes (L. Hansson, L. Fahrig \& G. Merriam, eds). Chapman \& Hall, London.

CAZETTA, E., RUBIM, P., LUNARDI, V.O., FRANCISCO, M.R. \& GALETTI, M. 2002. Frugivoria e dispersão de sementes de Taluma ovata (Magnoliaceae) no sudeste brasileiro. Ararajuba 10(2):199-206.
CORDEIRO, N.J. \& HOWE, H.F. 2003. Forest fragmentation severs mutualism between seed dispersers and an endemic African tree. Proc. Natl. Acad. Sci. 100(24): 14052-14056.

COSTA, C.M.R. 1998. Biodiversidade em Minas Gerais: um atlas para a sua conservação. Fundação Biodiversitas, Belo Horizonte.

DEVELEY, P. Métodos com estudos com aves 2003. In Métodos de estudos em biologia da conservação e manejo da vida silvestre (UNIVERSIDADE FEDERAL DO PARANÁ - UFPR, ed.). Fundação O Boticário de Proteção à natureza, Paraná, p. 153-179.

DEVELEY, P. \& ENDRIGO, E. 2004. Aves da Grande São Paulo. Aves e Fotos Editora, São Paulo.

EWERS, R.M. \& DIDHAM, R.K. 2006. Confounding factors in the detection of species responses to habitat fragmentation. Biol. Rev. 81(1):117-142.

FEELEY, K.J. \& TERBORGH, J.W. 2006. Habitat fragmentation and effects of herbivore (howler monkey) abundances on bird species richness. Ecology 87(1):144-150.

FONSECA, Y.F. \& ANTUNES, Z.A. 2007. Frugivoria e predação de sementes por aves no Parque Estadual Alberto Löfgren, São Paulo, SP. Rev. Inst. Flor. 19(2):81-91.

FUNDAÇÃO SOS MATA ATLÂNTICA \& INSTITUTO NACIONAL DE PESQUISAS ESPACIAIS. 2009. Atlas dos remanescentes florestais da Mata Atlântica. São Paulo.

GALETTI, M., PIZO, M. \& MORELLATO, P.C. 2003. Fenologia, frugivoria e dispersão de sementes. In Métodos de estudos em biologia da conservação e manejo da vida silvestre (L.J. Cullen, R. Rudran \& C.V. Padua, eds). Fundação O Boticário de Proteção à Natureza, Curitiba, p. 396-409.

GALETTI, M. \& PIZO, M.A. 1996. Fruit eating by birds in a forest fragment in southeastern Brazil. Ararajuba 4(2):71-79.

GARCIA, D. \& CHACOFF, N.P. 2006. Scale: dependent effects of habitat fragmentation on hawthorn Pollination, frugivory, and seed predation. Conserv. Biol. 21(2):400-411.

GUIMARÃES, M.A. 2003. Frugivoria por aves em Tapirira guianensis (Anacardiaceae) na zona urbana do município de Araruama, estado do Rio de Janeiro, sudeste brasileiro. Atual. Ornitol. (116):12-22.

HASUI, É. 1994. O papel das aves frugívoras na dispersão de sementes em um fragmento de floresta estacional semidecídua secundária, em São Paulo, SP. Dissertação de Mestrado, Universidade de São Paulo, São Paulo.

HERRERA, C.M. 2002. Seed dispersal by vertebrates. In Plant-animal interactions: an evolutionary approach (C.M. Herrera \& O. Pellmyr, eds). Blackwell Publishing, Malden, p. 185-208.

JORDANO, P., GALETTI, M., PIZO, M.A. \& SILVA, W.R. 2006. Ligando frugivoria e dispersão de sementes à biologia da conservação. In Biologia da conservação: essências (C.F.D. Rocha, H.G. Bergallo \& M.A.S. Alves, eds). Rima, São Carlos, p. 411-436.

KIRIBA, J.M., BLEHER, B., GAESE, K.B., CHIRA, R. \& FARWIG, N. 2008. Fragmentation and local disturbance of forests reduce frugivore diversity and fruit removal in Ficus thonningii trees. Bas. Appl. Ecol. 9(6):663-672.

KREBS, C.J. 1989. Ecological methodology. Harper \& Row, New York.

KRUGEL, M.M., BURGER, M.I. \& ALVES, M.A. 2006. Frugivoria por aves em Nectandra megapotamica (Lauraceae) em uma área de Floresta Estacional Decidual no Rio Grande do Sul, Brasil. Iheringia, Ser. Zool. 96(1):17-24.

LORENZI, H. 2000. Árvores brasileiras: manual de identificação e cultivo de plantas arbóreas nativas do Brasil. 3 ed. Instituto Plantarum, Nova Odessa, p. 168.

MACHADO, L.O.M. \& ROSA, G.A.B. 2005. Frugivoria por aves em Cytharexyllum myrianthum cham (Verbenaceae) em áreas de pastagens de Campinas, SP. Ararajuba 13(1):113-115.

MCGARIGAL, K., MARKS, B.J., HOLMES, C. \& ENE, E. 2002. FRAGSTATS: Computer software program produced by the authors at the University of Massachusetts, Amherst. www.umass.edu/landeco/research/ fragstats/fragstats.html (último acesso em 10/11/2008). 
MORAN, C., CATTERALL, C.P. \& KANOWSKI, J. 2008. Reduced dispersal of native plant species as a consequence of the reduced abundance of frugivore species in fragmented rainforest. Biol. Cons. 142(3):541-552.

MUSTAJARVI, K., SIIKAMÄKI P., RYTKÖNEN, S. \& LAMMI, A. 2001. Consequences of plant population size and density for plant: pollinator interactions and plant performance. J. Ecol. 89(1):80-87.

OLIVEIRA, R.L.S. \& FERREIRA, M.F. 2008. Unidades de paisagem da região de Alfenas, sul de Minas Gerais, a partir de dados do radar SRTM e imagem orbital ETM + LANDSAT 7, utilizando a abordagem geossistêmica. In I Semana de Geotecnologias. (CENTRO DE ANÁLISE E PLANEJAMENTO AMBIENTAL - CEAPLA, ed.) Rio Claro. http://200.145.36.250/ocs/index.php/ceapla/geotec/paper/view/34/2 (último acesso em 15/12/2008).

OZINGA, A.W. 2004. Dispersal potential in plant communities depends on environmental conditions. J. Ecol. 92(5):767- 777.

PASCOTTO, M.C. 2006. Avifauna dispersora de sementes de Alchorna glandulosa (Euphorbiaceae) em uma área de mata ciliar no estado de São Paulo. Rev. Bras. Ornitol. 14(4):291-296.

PEDRONI, F., SANCHEZ, M. \& SANTOS, F.A.M. 2002. Fenologia da copaíba (Copaifera langsdorffii Desf. - Leguminosae, Caesalpinioideae) em uma floresta semidecídua no sudeste do Brasil. Rev. Bras. Bot. 25(2):183-194.

PINESCHI, R.B. 1990. Aves como dispersores de sete espécies de Rapanea (Myrsinaceae) no Maciço do Itatiaia, Estados do Rio de Janeiro e Minas Gerais. Ararajuba 1(1):73-78.

PIZO, A.M. 2004. Frugivory and habitat use by fruit: eating birds in a fragmented landscape on southeast Brazil. Ornitol. Neotrop. 15(suppl.):117-126.

PIZO, A.M. \& VIEIRA, E.M. 2004. Palm harvesting affects seed predation of Euterpe edulis, a threatened palm of the Brazilian Atlantic Forest. Braz. J. Biol. 64(3b):1-8.

PIZO, A.M. \& VIEIRA, E.M. 2004. Granivorous birds as potentially important post: dispersal seed in a Brazilian Forest Fragment. Biotropica 36(3):417-423.

PRICE, O.F., WOINAESKI, J.C.Z. \& ROBINSON, D. 1999. Very large area requirements for fruvivorous birds in monsoon rainforests of the Northern Territory, Australia. Biol. Cons 91(2-3):169-180.
PRIMACK, B.R. \& RODRIGUES, E. 2001. Biologia da conservação. Editora Planta, Londrina, p. 69-133.

RAMOS, F.N., JOSÉ, J., SOLFERINI, V.N. \& SANTOS, F.A.M. 2007. Quality of seeds produced by Psychotria tenuinervis (Rubiaceae): distance from anthropogenic and natural edges of Atlantic forest fragment. Bioch. Genet. 45(5-6):441-458.

RIBON, R., SIMON, J.E. \& MATTOS, G.T. 2003. Bird extinctions in Atlantic Forest fragments of Viçosa region, southeastern Brazil. Cons. Biol. 17(6):1827-1839.

SANTOS, B.A. 2004. Influência da distância geográfica na similaridade florística de clareiras e sub-bosques em uma floresta de terra firme na Amazônia Central. Editora do INPA, Manaus. http://74.125.95.132/ search?q=cache:http://tamandua.inpa.gov.br/ pdbff/cursos/efa/ livro/2004/PDFs/41_final/braulio.pdf (último acesso em 11/10/2008).

SICK, H. 2001. Ornitologia brasileira. 2 ed. Nova Fronteira, Rio de Janeiro.

SPIEGEL, O. \& NATHAN, R. 2007. Incorporating dispersal distance into the disperser effectiveness framework: frugivorous birds provide complementary dispersal to plants in a patchy environment. Ecol. Lett. 10(8):718-728.

STOTZ, D.F., FITZPATRICK, J.W., PARKER III, T.A. \& MOSKOVITZ, D.K. 1996. Neotropical birds: ecology and conservation. University of Chicago Press, Chicago.

VALDIVIA, C.E. \& SIMONETTI, J.A. 2007. Decreased frugivory and seed germination rate do not reduce seedling recruitment rates of Aristotelia chilensis in a fragmented forest. Biodiversis Conserv. 16(6):15931602.

WATSON, J.E.M., WHITTAKER, R.J. \& DAWSON, T.P. 2004. Avifaunal responses to habitat fragmentation in the threatened littoral forests of south-eastern Madagascar. J. Biogeo. 31(11):1791-1807.

Recebido em 09/07/09

Versão reformulada recebida em 18/12/09

Publicado em 14/01/10 